\title{
USO DE LA TÉCNICA DE MIOFEEDBACK ORIENTADA EN ACTIVIDADES DE LA VIDA DIARIA BÁSICAS EN PERSONAS SECUELADAS DE UN ACCIDENTE CEREBRO VASCULAR.
}

\section{Myofeedback In Basic Activities Daily Life In Patiens With Stroke}

\author{
Daniela Salgado $\mathrm{J}^{\mathrm{i}}$, Maricel Garrido $\mathbf{M}^{\text {ii }}$, Evelyn Alvarez $^{\mathrm{iii}}$. \\ Colaboran: Sebastián Vergara, Sebastián, Luis González, Pamela Salcidua, Sebastián
}

Tobar. (estudiantes en práctica)

\section{RESUMEN}

El Accidente Cerebro Vascular (ACV) es la segunda causa de muerte en Chile y sus secuelas repercuten en el desempeño ocupacional; 50\% necesita asistencia para bañarse, $30 \%$ para vestirse y $10 \%$ para higiene personal. La recuperación funcional e independencia es el objetivo de la rehabilitación, sin embargo, existe escasa evidencia de técnicas efectivas para usuarios secuelados de un ACV.

El Miofeedback se ha dirigido al reaprendizaje de movimientos aislados y repetitivos, por lo tanto este trabajo tiene como objetivo "conocer el funcionamiento motor, ocupacional y de satisfacción de movimientos en usuarios con ACV antes y después de la aplicación de un Programa de Miofeedback orientado en AVDB”.

El grupo de estudio corresponde 8 personas. Cada uno tuvo 10 sesiones individuales de 60 minutos, 2 veces por semana, usando el miofeedback durante la ejecución de las AVDB. Evaluadores doble enmascarado aplican 5 pautas de evaluación al inicio y al término del Programa.

Los participantes logran mejoras estadísticamente significativas en 5 de las 6 pautas, utilizando una prueba no paramétrica (t-student)..Según estos resultados el uso de Miofeedback genera cambios significativos en los participantes, permitiendo generar evidencia tanto del uso de la ocupación como medio terapéutico, como de la tecnología al servicio de la rehabilitación.

Palabras claves: Accidente cerebro vascular, miofeedback, rehabilitación motora, actividades de la vida diaria básica.

\section{ABSTRACT}

The Stroke is the second reason of death in Chile and its sequels (motor, cognitive, etc) affect the occupational performance, causing dependence in basic activities of the daily living (BADL): 50\% needs assistance to washing, 30\% to dress and 10\% for personal hygiene. The functional recovery and independence is the aim of the rehabilitation, but there exists scanty evidence of effective techniques for chronic users whit stroke.

The myofeedback has gone to the relearning of aislated and repetitive movement, therefore this work has as aim "to know the motor occupational function and satisfaction of 
movement of stroke patients before and after the application of a Miofeedback's Program, oriented to BADL"

The group of study corresponds to 8 persons. Each one had 10 individual sessions of 60 minutes, twice a week, using the Miofeedback during the performance of the BADL. Double blinded apply 5 test; motor, occupational function and satisfaction of movement to initial and final of treatment.

Participants achieve statistically significant improvements in 5 of 6 tests, using nonparametric test (t-student). According to these results Miofeedback's use generates significant changes in the participants, allowing to generate evidence both on the use of occupation as a therapeutic means, as on the technology at the service of rehabilitation

Key Word: Stroke, Myofeedback, Motor Rehabilitation, ADL.

\footnotetext{
${ }^{\text {i }}$ Terapeuta Ocupacional, Universidad de Chile. Licenciada en Ciencia de la Ocupación. Hospital Clínico Universidad Católica. danielita_pia@hotmail.com

ii Terapeuta Ocupacional, Universidad de Chile. Licenciada en Ciencia de la Ocupación. Hospital Clínico Universidad de Chile. maricel.garrido.m@gmail.com

iii Terapeuta Ocupacional, Universidad de Chile. Licenciada en Ciencia de la Ocupación. Magíster (C) Neurociencia. Hospital Clínico Universidad de Chile. Universidad de Chile. evalvarez@med.uchile.cl.
}

\section{INTRODUCCIÓN}

El accidente cerebro vascular (ACV) es la segunda causa específica de muerte en Chile y la primera causa de hospitalizaciones sobre los 65 años. De los usuarios sobrevivientes a los 6 meses, sólo un 37\% lo hacen de manera independiente y un 63\% necesita algún tipo de ayuda $^{1}$; lo que significa un importante impacto económico y social para la familia y el país. Estas alteraciones generan un impacto en el desempeño ocupacional de los usuarios, imposibilitándolos de cumplir las expectativas de su rol y las demandas de las actividades que realizaban hasta antes del accidente; en este sentido, las actividades de la vida diaria (AVD) también se ven afectadas ${ }^{2}$, entendiéndolas como las actividades orientadas hacia el cuidado del propio cuerpo y consideradas ocupaciones al presentar forma ${ }^{\mathrm{I}}$, función ${ }^{\text {II }} \mathrm{y}$ significado III (Nota al pie) para cada persona. En el caso de usuarios secuelados por un ACV: el $50 \%$ requiere asistencia para bañarse, el 30\% para vestirse y el $10 \%$ para higiene personal ${ }^{3}$.

IForma: se refiere a los aspectos de las ocupaciones que son directamente observables.

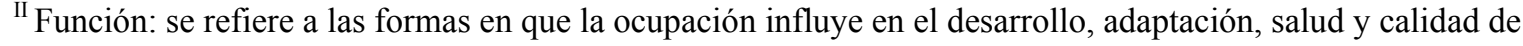
vida.

III Significado: es el valor personal o individual que se da a las actividades. 
Si bien es cierto, se observa un gran incremento e impacto de esta patología, en la actualidad existe limitada evidencia experimental disponible, indicando qué técnicas de rehabilitación son efectivas para usuarios crónicos secuelados de un ACV; por lo que se hace necesario sistematizar las experiencias y estandarizar protocolos de intervención para demostrar la real eficacia de estos procedimientos. Es por eso que surge esta investigación, enfocada en el uso del Miofeedback, un aparato que hace consciente la actividad muscular (con señales visuales y auditivas) a través de electrodos que miden la actividad eléctrica ${ }^{4}$.

Este aparato tecnológico ha mostrado beneficios en la fuerza, rango de movimiento, tono muscular, funcionamiento de brazo y mano, en movimientos específicos y repetitivos, se considera una herramienta de reeducación muscular en usuarios secuelados de un ACV en condiciones crónica $^{5-9}$, se debe mencionar que la metodología utilizada en estos estudios no permite refutar o apoyar el uso de la técnica. Por otra parte en la práctica de la Terapia Ocupacional, es primordial la recuperación e independencia de las AVD, la realización de estas actividades es concordante con evidencia actual que plantea la importancia de la motivación de los sujetos en el proceso de recuperación ${ }^{10} \mathrm{y}$ la consideración del contexto, ya que genera mayor activación cortical ${ }^{11}$.

Debido a estas razones, ésta investigación se orienta a conocer la práctica del Miofeedback durante la ejecución de las AVD Básicas; surgiendo la pregunta, si la ¿utilización del Miofeedback en una AVDB significativa, incrementan el funcionamiento motor, ocupacional y satisfacción de movimientos de los participantes? Para responder a dicha pregunta se realizaron evaluaciones de funcionamiento motor, ocupacional y de satisfacción de los movimientos, antes y después de un tratamiento de 10 sesiones a personas secueladas de un ACV atendidas en el Hospital Clínico José Joaquín Aguirre.

\section{METODOLOGIA}

Para ingresar al estudio los pacientes debían tener entre 18 y 70 años, un diagnóstico de ACV con un mínimo de 6 meses y un máximo de 30 meses de evolución, tener sólo un evento de $\mathrm{ACV}$, presentar en el hemicuerpo afectado por lo menos $20^{\circ}$ de extensión de 
muñeca y $10^{\circ}$ de extensión metacarpofalángica. Los criterios de exclusión fueron presentar alteraciones sensoriales, perceptivas o cognitivas, patología psicoafectiva sin tratamiento, hombro doloroso o subluxación de hombro. A todos los sujetos se les informó las características del estudio, al que accedieron voluntariamente. (condiciones consentimiento informado)

La muestra obtenida finalmente corresponde a 6 personas de ambos sexos ( 5 hombres y 1 mujer) que presentan el diagnóstico de ACV isquémico con un mínimo de 6 meses y un máximo de 30 meses de evolución, que presentan secuelas motoras. Todos los pacientes participantes fueron atendidos en la Unidad de Tratamiento del Ataque Cerebro Vascular (UTAC) del Hospital José Joaquín Aguirre durante el año 2007. El número de participantes fue calculado para obtener una confiabilidad $95 \%$, considerando el número de ingresos de pacientes al año en la UTAC.

Evaluadores doble enmascarados aplicaron evaluaciones de funcionamiento ocupacional, motor y satisfacción de movimientos al inicio y al término del Programa. Además, en cada sesión se registraron los niveles de contracción máxima en el grupo muscular seleccionado para cada usuario. Las pautas utilizadas según tipo de funcionamiento fueron':

Funcionamiento Ocupacional: Katz, Barthel, Lawton \& Brody.

Funcionamiento Motor: Test Action Research Arm (funcionamiento de miembro superior).

Satisfacción de movimientos: Medida Canadiense de Desempeño Ocupacional, autopercepción del funcionamiento motor en actividades cotidianas (APDM) en cantidad y calidad.

\footnotetext{
${ }^{\text {I }}$ Katz, Barthel son evaluaciones que miden actividades de la vida diaria básica, Lawton \& Brody evalúa las las actividades de la vida diaria instrumentales, Test action research arm evalúa el funcionamiento del miembro superior afectado en actividades de agarre, prehensión cilíndrica y movimiento grueso. Medida Canadiense de Desempeño Ocupacional evalúa el cambio autopercibido en los problemas de desempeño ocupacional a lo largo del tiempo. Pauta de autopercepción del funcionamiento motor en actividades cotidianas (APDM), entrevista semiestructurada acerca de cómo los usuarios utilizan su extremidad afectada en 30 AVD durante la semana anterior a la entrevista.
} 
Como protocolo de intervención se efectuó la elección de actividades de la vida diaria básicas por los usuarios, se consideraron actividades bimanuales que promuevan el uso de lado afectado y actividades que promuevan utilización de musculatura gravitatoria del miembro superior, en este último punto la musculatura que cumple esta característica, corresponde a los abductores de hombro, rotadores externos de hombro, extensores de codo, supinadores de antebrazo; extensores de muñeca, dedos y pulgar; abductores de dedos y pulgar.

Se planteó a cada usuario un listado de actividades (tabla 1), de la cual ellos seleccionaron una según la motivación y significancia que tuviera para ellos, además que en el momento de la evaluación no la logre realizar adecuadamente.

Tabla $N^{\circ} 1$ : AVDB posibles e trabajar.

\begin{tabular}{|l|l|}
\hline AVD & Actividad \\
\hline 1.-Baño, ducha & Jabonar el cuerpo \\
\hline & Secar el cuerpo \\
\hline 2.-Vestuario & Poner y sacar una chaqueta \\
\hline & Poner y sacar calcetines \\
\hline & Poner el sostén \\
\hline 3.-Alimentación & Preparar un pan \\
\hline & Uso de tenedor o cuchara \\
\hline & Tomar líquido de un vaso \\
\hline 4.-Higiene personal y aseo & Partir una marraqueta y comer \\
\hline & Lavar la cara \\
\hline & Lavar el pelo \\
\hline & Aplicar crema en el cuerpo \\
\hline 5.-Higiene en el inodoro & Aplicar desodorante \\
\hline
\end{tabular}

Se realizaron 10 sesiones individuales de 60 minutos, 2 veces por semana. Donde cada sesión se estructuro de la siguiente manera: 
1.Acondicionamiento del Usuario: Preparar el segmento corporal, para la instalación de los electrodos de registro en el grupo muscular a trabajar.

2.Entrenamiento de AVD con uso de Miofeedback: realización de la actividad de la vida diaria básica, elegida por el usuario. Durante el movimiento el usuario recibe la retroalimentación visual o auditiva de su funcionamiento.

3.Actividades de AVDB sin Miofeedback: el usuario practica las AVDB sin el aparato de miofeedback.

4.Relajación del segmento ejercitado.

Para el análisis general de la muestra y los cambios en las contracciones musculares, se realizó estadística descriptiva, y para evaluar el impacto de la técnica se analizaron las pautas de evaluación con la medida de distribución de probabilidad t-Student ${ }^{\mathrm{I}}$.

\section{RESULTADOS}

A continuación se muestran los resultados obtenidos en esta investigación, comenzando con la descripción de la muestra en estudio, seguido por un análisis tanto de los datos entregados por el Miofeedback como por los tipos de funcionamiento.

\section{1.- Características de la Muestra y AVDB seleccionadas}

El grupo de estudio corresponde a 6 personas, 2 mujeres y 4 hombres. Con un promedio y desviación estándar de edad de 37,6 \pm 17,17 y 12,5 \pm 1,7 años de estudio. Sólo una de las personas de la muestra ha mantenido su actividad productiva post $\mathrm{ACV}$, los demás no realizan ningún tipo de actividad productiva. Todos presentaron un ACV de tipo isquémico, con un promedio de $14,8 \pm 7,8$ meses de tiempo secuelar. Todos los pacientes asistieron a terapia kinesiológica además del programa de Miofeedback (tabla 2).

\footnotetext{
${ }^{\mathrm{I}}$ Es una distribución de probabilidad que estima el la diferencias entre dos medias muestrales.
} 
Las AVDB más seleccionadas por los usuarios fueron las de vestuario (poner calcetines, abotonar) y alimentación (preparar pan, cortar carne).

Tabla $N^{\circ} 2:$ Características generales de la muestra.

\begin{tabular}{|c|c|c|c|c|c|c|}
\hline & $\begin{array}{l}\text { Usuario } \\
\text { N'1 }\end{array}$ & $\begin{array}{l}\text { Usuario } \\
\text { N'2 }\end{array}$ & $\begin{array}{l}\text { Usuario } \\
\text { N'3 }\end{array}$ & $\begin{array}{l}\text { Usuario } \\
\text { N'4 }\end{array}$ & $\begin{array}{l}\text { Usuario } \\
\text { N'5 }\end{array}$ & $\begin{array}{l}\text { Usuario } \\
\text { N'6 }\end{array}$ \\
\hline Edad & 48 & 37 & 35 & 17 & 22 & 65 \\
\hline $\begin{array}{l}\text { Años de } \\
\text { estudio }\end{array}$ & 12 & 12 & 12 & 11 & 12 & 16 \\
\hline $\begin{array}{l}\text { Actividad } \\
\text { laboral } \\
\text { previa }\end{array}$ & $\begin{array}{l}\text { Dueña de } \\
\text { casa. }\end{array}$ & Metalúrgico & Cocinero & No. & $\begin{array}{l}\text { Operario } \\
\text { computador. }\end{array}$ & $\begin{array}{l}\text { Escritora } \\
\text { libros de } \\
\text { matemáticas. }\end{array}$ \\
\hline $\begin{array}{l}\text { Actividad } \\
\text { laboral } \\
\text { actual }\end{array}$ & $\begin{array}{l}\text { Dueña de } \\
\text { casa. }\end{array}$ & No. & No. & No. & No. & No. \\
\hline $\begin{array}{l}\text { Tiempo } \\
\text { secuelar }\end{array}$ & 8 meses. & 9 meses. & $\begin{array}{l}1 \text { año } 8 \\
\text { meses. }\end{array}$ & $\begin{array}{l}1 \text { año } 2 \\
\text { meses. }\end{array}$ & 10 meses. & $\begin{array}{l}2 \text { años } 4 \\
\text { meses. }\end{array}$ \\
\hline $\begin{array}{l}\text { Tipos de } \\
\text { ACV }\end{array}$ & $\begin{array}{l}\text { Isquémico } \\
\text { Izquierda }\end{array}$ & $\begin{array}{l}\text { Isquémico } \\
\text { derecho }\end{array}$ & $\begin{array}{l}\text { Isquémico } \\
\text { Izquierda }\end{array}$ & $\begin{array}{l}\text { Isquémico } \\
\text { Derecho }\end{array}$ & $\begin{array}{l}\text { Isquémico } \\
\text { Derecho }\end{array}$ & $\begin{array}{l}\text { Isquémico } \\
\text { Izquierda }\end{array}$ \\
\hline Dominancia & Diestra & Diestra & Diestra & Diestra & Diestra & Diestra \\
\hline $\begin{array}{l}\text { Terapias } \\
\text { Asociadas }\end{array}$ & Kinesiología & Kinesiología & $\begin{array}{l}\text { Kinesiología } \\
\text { Fonoaudiolog } \\
\text { ía }\end{array}$ & Kinesiología & Kinesiología & Kinesiología \\
\hline
\end{tabular}

\section{$\underline{\text { Resultados del Miofeedback }}$}

La tabla $\mathrm{N}^{\circ} 3$ muestra los resultados promedios y las desviación estándar de las contracciones musculares máximas (medidas por el miofeedback) obtenidas al inicio y al término del programa por cada uno de los usuarios. La variación promedio de los seis usuarios fue altamente significativa $(\mathrm{P}<0,005)$, reflejo de una mayor activación de la musculatura gravitatoria seleccionada. 
Tabla Nº: Resultados Generales del Miofeedback.

\begin{tabular}{|l|l|l|l|}
\hline Usuario & $\overline{\mathbf{x}} \pm$ Desvest & $\overline{\mathbf{x}} \pm$ Desvest & Variación \\
\hline Usuario 1 & $26.2 \pm 9.36$ & $62.13 \pm 7.21$ & 35.93 \\
\hline Usuario 2 & $14.17 \pm 5.67$ & $68.17 \pm 16.2$ & 54 \\
\hline Usuario 3 & $14 \pm 1.41$ & $38.98 \pm 0.03$ & 24.98 \\
\hline Usuario 4 & $53.54 \pm 1.52$ & $90.77 \pm 5.62$ & 37.23 \\
\hline Usuario 4 & $55.65 \pm 9.16$ & $92.43 \pm 8.11$ & 36.78 \\
\hline Usuario 5 & $48.025 \pm 7.82$ & $99.52 \pm 0.75$ & 51.5 \\
\hline Usuario 6 & $44.4 \pm 4.16$ & $74.9 \pm 2.98$ & 30.5 \\
\hline & Variación promedio & $\mathbf{3 8 . 7 * *}$ \\
\hline
\end{tabular}

** $\mathbf{P}<0,005$

Los datos entregados por el Miofeedback, demuestran que todos los usuarios mostraron grandes avances en relación al movimiento de la musculatura gravitatoria trabajada, obteniéndose un resultado final más alto en los usuarios más jóvenes y con mejor activación muscular inicial (usuario $\mathrm{N}^{\mathrm{o}} 4$ y 5).

El aumento en la activación de la musculatura trabajada, no tuvo una relación directa con la funcionalidad del miembro superior afectado en todos los usuarios; observándose mayores avances en aquellos usuarios que presentaron mejores resultados en las evaluaciones iniciales (usuario 2,4 y 5); logrando incorporar el hemicuerpo afectado en actividades bimanuales, mejorando los patrones de movimientos, logrando disociación de movimientos finos y disminuyendo las compensaciones.

En los usuarios en que su nivel inicial de funcionamiento fue bajo (usuarios 1,3 y 6), igualmente lograron avances con el programa de miofeedback; pero logros sólo se enfocaron a una mayor incorporación del hemicuerpo afectado como apoyo en diversas actividades. 


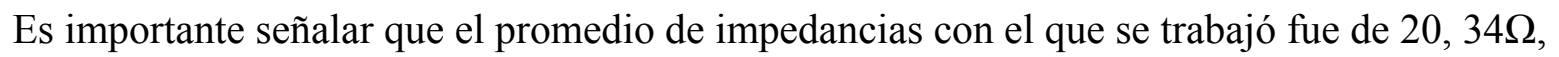
considerado como una baja resistencia de la piel a la conducción en el Miofeedback; otorgando datos fidedignos de las señales musculares.

\section{$\underline{\text { Resultados de las Pautas de Evaluación }}$}

La presente tabla muestra los resultados promedios y la desviación estándar iniciales y finales de cada pauta de evaluación, donde se encontró cambios significativamente estadísticos (t-student) en 5 de las 6 pautas.

Tabla Nº: Resultados según pautas de Evaluación

\begin{tabular}{|l|l|l|}
\hline \multicolumn{2}{|c|}{ Tabla Resultados Pautas de Evaluación } \\
\hline Pautas & $\bar{x}$ i \pm Desvest & $\overline{x f} \pm$ Desvest \\
\hline Índice de Katz & $6,5 \pm 0,55$ & $6,83 \pm 0,41$ \\
\hline Índice de Barthel & $76,67 \pm 9,83$ & $95 \pm 10^{* *}$ \\
\hline Escala de Lawton y & $2,80 \pm 1,64$ & $5,5 \pm 1,87^{*}$ \\
\hline Brody & & \\
\hline Action Research Arm & $13,17 \pm 18,07$ & $29,17 \pm 25,61^{*}$ \\
\hline APDM (Cantidad) & $10,5 \pm 7,06$ & $16,5 \pm 5,32^{* *}$ \\
\hline APDM (Puntaje) & $26,5 \pm 24,67$ & \\
\hline COPM (desempeño) & $3,33 \pm 1,67$ & $6,42 \pm 0,79 *$ \\
\hline COPM (satisfacción) & $3,17 \pm 1,47$ & $6,92 \pm 1,08^{*}$ \\
\hline
\end{tabular}




\section{Funcionamiento Ocupacional}

Katz es la única pauta que no muestra avances significativos, debido a su bajo nivel de sensibilidad. Se obtiene un promedio de $6,83 \pm 0,41$ equivalente a la categoria B (independencias en todas, menos en una).

Los resultados obtenidos en el índice de Barthel son altamente significativos $(\mathrm{P}<0,005)$, reflejando mayor independencia de los usuarios; esto expresa finalmente un promedio de $95 \pm 10$ de los 100 puntos para la independencia completa. En la evaluación inicial 1 de los usuarios era independiente y luego del programa esto subió a 4 pacientes. En la escala de evaluación de AVDI (Lawton y Brody), también se obtuvieron diferencias significativas en los resultados $(\mathrm{P}<0,05)$, aspecto no esperado en los objetivos iniciales.

\section{Funcionamiento Motor}

Medido con la pauta ARA, la cual entrega resultados en relación a la movilidad del miembro superior afectado; se obtuvo en la evaluación previa a la intervención un total $13,17 \pm 18,07$, en cambio en la evaluación final de la intervención alcanzó un promedio de $29,17 \pm 25,61$ de los 57 puntos esperados, logrando diferencias estadísticas significativas para esta pauta $(\mathrm{P}<0,05)$. Al no tener una clasificación general, no hay categorizaciones en relación al puntaje, solo en el desglose de la pauta en si; se obtuvo en la evaluación final un promedio de 4,83 de18 puntos para agarre, 4,67 de un total de 12 puntos para prehensión cilíndrica, en las pinzas se obtuvo 4 de 18 y en los movimientos gruesos 4 puntos de un total 17 puntos.

\section{Satisfacción de Movimientos}

Según la pauta de autopercepción del funcionamiento motor en actividades cotidianas, se obtuvo al final de la intervención un promedio de $16,5 \pm 5,32$ de un total de 30 puntos en relación a la cantidad (nivel de frecuencia) de la actividad desarrollada; la mayoría de las actividades mencionadas en la pauta, no podían ser realizadas antes del programa y luego de la intervención los usuarios refieren que si pudieron realizarlas. Los resultados 
obtenidos en esta pauta son altamente significativos $(\mathrm{P}<0,005)$. Además los sujetos mencionan un incremento importante en la incorporación del lado afectado en las actividades cotidianas.

Por otra parte, al evaluar el desempeño y la satisfacción de los usuarios en relación a las AVDB escogidas (con la evaluación COPM); se obtienen resultados altamente significativos $(\mathrm{P}<0,005)$; lo cual es considerablemente positivo ya que hace referencia a la autopercepción de las habilidades iniciales y finales de los usuarios en relación a las AVDB escogidas y los avances logrados en ellas.

\section{DISCUSIÓN.}

El ACV es una patología de gran incidencia en nuestro país ${ }^{1}$, que en éste estudio integró a sujetos más jóvenes de lo esperado, ya que según estudios, la incidencia de ACV aumenta $10 \%$ por año después de los 45 años, y la posibilidad de tener un ACV es más del doble por cada década de vida después de los 55 años ${ }^{12}$. Esto implica, que la mayoría de los usuarios se encuentra en plena etapa de productividad, por lo tanto, el rol laboral se ve altamente afectado, teniendo dificultades en retomar su trabajo previo debido a las secuelas generadas por el $\mathrm{ACV}^{13}$. La muestra del estudio es un reflejo de esto, ya que 4 de los participantes no han podido retomar su rol productivo, con el consecuente impacto personal, familiar y social.

EL ACV generalmente produce daños, en mayor o menor grado, en el feedback sensorial necesario para el control y aprendizaje motor ${ }^{14}$, esto limita la capacidad de los usuarios, para adquirir, modificar o ajustar habilidades, ya que no perciben de manera correcta, cual es el funcionamiento real de su cuerpo, ya que "los movimientos se ajustan de acuerdo a la percepción”, según la Teoría de Circuito Cerrado- Close Loop-de Adams ${ }^{15}$. En la presente investigación, la amplificación de las señales musculares (miofeedback), a través de estímulos visuales-auditivo-somatosensoriales, compensa el feedback deficitario de los usuarios, optimizando el aprendizaje motor, lo cual es apoyado desde la Teoría de Esquemas de Schmidt, que plantea que mientras más retroalimentación se entregue, más se 
reforzaran los esquemas ${ }^{14}$. En términos generales el Miofeedback presenta ventajas, ya que al entregar retroalimentación inmediata, genera mayor motivación de los usuarios en el uso de la misma y en la posibilidad de ir superándose; lo cual favorece la adherencia al tratamiento; por lo que dentro de la investigación; la retroalimentación, así como la motivación juegan un papel fundamental en la readquisición de habilidades, lo que puede ser un aporte a los tratamientos en general, tomando estos dos factores como premisas en los tratamientos de rehabilitación en general. Una desventaja del uso de la técnica, es que se debe cumplir con ciertas normas que enlentecen las sesiones de tratamiento.

Dentro de las áreas investigadas en el estudio, a nivel de funcionamiento ocupacional, no se encontraron diferencias significativas en el Índice de Katz, lo cual puede deberse a la falta de sensibilidad de la pauta debido a que presenta categorías muy generales de evaluación; observándose mínimos avances en la independencia de las AVDB. Estos datos se contrarrestan con los obtenidos por la pauta del Índice de Barthel, la cual a pesar de evaluar la misma área, es más sensible, por lo que los datos finales sí fueron significativos, donde los hombres alcanzaron la puntuación máxima (100 puntos), que equivale la independencia completa en AVDB. Además se debe destacar que los pacientes presentaron evaluaciones iniciales con un nivel de independencia leve, y después de la intervención un $65 \%$ de los sujetos alcanzaron niveles de independencia completa.

En las AVDI (Escala de Lawton y Brody) los pacientes obtuvieron avances estadísticamente significativos, a pesar que esto no era esperado, ya que la intervención esta centrada en las AVDB. Sin embargo, este aumento puede ser reflejo de la capacidad de extrapolar las habilidades adquiridas con el programa a actividades de mayor complejidad, como en preparación de alimentos, cuidado de la casa y realización de compras. La actividad de lavado de ropa, no obtuvo avances, lo que se puede deber al contexto cultural chileno, donde esta actividad es realizada principalmente por mujeres o porque esta actividad no ha sido realizada previamente.

En el Funcionamiento Motor se encontraron avances significativos en los subitems de agarre y prensión cilíndrica de la prueba de Action Research Arm, estos movimientos se 
relacionan directamente en AVDB que utilizan implementos: uso de cubiertos y tomar un vaso en alimentación, tomar utensilios de aseo (jabón, shampoo) o simplemente la utilización de la mano afectada como apoyo en actividades bimanuales. Otra información obtenida a través del reporte de los pacientes es que lograron obtener una mayor conciencia y control sobre el estado de tensión (tono) muscular de la extremidad superior afectada; por lo tanto, sería útil cuantificar en futuras investigaciones al inicio y al final, el tono muscular utilizando la escala de Asworth, ya que el uso del Miofeedback generaría un impacto importante en esta condición, sensible de medir con esta pauta.

En la satisfacción de los movimientos, los sujetos perciben que aumentaron la cantidad del uso del hemicuerpo afectado, lo que refleja un incremento importante en la incorporación del lado afectado en las actividades cotidianas. A esto se suma el aumento sustancial en la percepción de calidad de movimiento expresada por los usuarios. Al ser una pauta que evalúa percepción, permite ser sensible en relación a los logros obtenidos por cada usuario y la visión que éste tiene de sus logros en su contexto diario; aspectos que otras pautas no evalúan al tener categorías rígidas y muchas veces amplias que no dan cuenta de la sutileza de dichos cambios.

Enfocar el tratamiento en AVDB generó en los usuarios la motivación por retomar la independencia o disminuir los tiempos en los que éstas se realizan, lo cual fue posible de observar de manera directa durante las sesiones, ya que los usuarios al trabajar durante una actividad motivadora y cotidiana, pusieron más énfasis en el logro de dicha actividad, que en el movimiento aislado de sus segmentos corporales, ayudados en un inicio por el feedback visual y auditivo, para al final de las sesiones realizarlo sin este apoyo. Este resultado es concordante con evidencia previa, donde pacientes con hemiplejía, presentaron importantes avances en cuanto a la recuperación del movimiento y funcionamiento ocupacional, después de una intervención basada en el juego ${ }^{10}$.

En resumen, el programa de Miofeedback orientado en una AVDB generó cambios estadísticamente significativos en todos los parámetros definidos: motor, ocupacional y nivel de satisfacción, además se observó un aumento progresivo de la contracción de la 
musculatura implicada; los datos obtenidos superaron lo esperado para la investigación, de acuerdo a lo reportado por la literatura. Además, la integración del brazo afectado y la posibilidad de generalizar los logros obtenidos en otras actividades que tuvieran dificultades similares a las AVDB escogida; es otro logro alcanzado. Esto se refleja en la alta independencia lograda en las pautas de Barthel, Katz y Lawton. Por otro lado, de acuerdo a lo referido por los usuarios, la técnica les brindó mayor seguridad en poder desarrollar o participar en distintas actividades y desempeñarse eficientemente en ellas.

Por último, esta investigación pretende valorar la integración de nuevas técnicas y uso de herramientas tecnológicas en el quehacer de los terapeutas ocupacionales para la rehabilitación de este tipo de patologías; ya que permiten conocer y disponer de información de los usuarios que sería difícil de obtener sin estos medios, haciendo más perceptibles los avances y siendo un importante agente motivador en las terapias.

\section{REFERENCIAS BIBLIOGRÁFICAS}

1. LAVADOS, P. Unidades de tratamiento del Ataque cerebrovascular en Chile. [en línea] Revista Médica de Chile, 2005;11:1271-1273. http://www.scielo.cl/scielo.php?pid=S003498872005001100001\&script=sci_arttext [consulta: 23 marzo 2008].

2. THE STROKE ASSOCIATION. http://www.stroke.org.uk/document.rm?id=877. The stroke statistics.

3. GARCÍA DOMINGO, A. M. Tratamiento de Terapia Ocupacional en el Accidente cerebro vascular. Edición No 3, Revista Gallega de Terapia ocupacional, Febrero de 2006.

4. BANGUERO, L. Herrera, Alejandro. Jaramillo, Roberto. sEMG para control de prótesis mioeléctricas. [en línea] Escuela de Ingeniería de Antioquia e Instituto de Ciencias de la Salud, 2006.

$<$ http://bioinstrumentacion.eia.edu.co/docs/signals/2006/exposiciones/sEMG_docu mento.pdf $>$. 
5. MORELAND J, THOMSON MA. Efficacy of electromyographic biofeedback compared with conventional physical therapy for upper-extremity function in patients following stroke: a research overview and meta-analysis. PHYS THER Vol. 74, No. 6, June 1994, pp. 534-543 .

6. -WOODFORD H, PRICE C. EMG biofeedback for the recovery of motor function after stroke. Cochrane Database Syst Rev. 2007 Apr 18;(2):CD004585.

7. CROW JL, LINCOLN NB, NOURI FM, DE WEERDT W. The effectiveness of EMG biofeedback in the treatment of arm function after stroke. Int Disabil Stud. 1989 Oct-Dec;11(4):155-60.

8. ARMAGAN O, TASCIOGLU F, ONER C. Electromyographic biofeedback in the treatment of the hemiplegic hand: a placebo-controlled study. Am J Phys Med Rehabil. 2003 Nov;82(11):856-61.

9. BRADLEY L, HART BB, MANDANA S. Electromyographic biofeedback for gait training after stroke. Clinical Rehabilitation. 1998 Feb;12(1):11-22.

10. S. WOOD, N. MURILLO, P. BACH-Y-RITA, R. LEDER, J. MARKS, S. Page. Motivating, Game-Based Stroke Rehabilitation: A Brief Report. Top Stroke Rehabil. 2003 Summer;10(2):134-40

11. M. IACOBONI y col. Grasping the intentions of others with one's own mirror neuron system. PLoS Biol. 2005 Mar;3(3):e79. Epub 2005 Feb 22.

12. DAVIS P, HACHINSKI V. Epidemiology of Cardiovascular isease. Neuroepidemiology: CRC Press 1991: 27-53.

13. D. PAPALIA. Desarrollo Humano. Editorial Mcgraw-hill, $9^{a}$ edición (2004).

14. SOTO, S.. Manual de Semiologia. 1ra Edición. Editorial Andrés Bello 1978.

15. WILLARD and SPACKMAN. Teorias de Control Motor. 10a Edición. Editorial Panamericana. 2003; 267-273. 